\title{
Determinación del perfil de resistencia antibiótica de Salmonella enterica aislada de cerdos faenados en un matadero de Lima, Perú
}

\author{
Determination of the profile of antibiotic resistance of Salmonella enterica \\ isolated from pigs in a slaughterhouse in Lima, Peru
}

\author{
Arnold Ríos C. ${ }^{1}$, Siever Morales-Cauti ${ }^{2}$, Miguel Vilca L. ${ }^{1}$, Andrea Carhuallanqui P. ${ }^{1}$ \\ Daphne Ramos D.,3
}

\section{Resumen}

El objetivo del presente trabajo fue determinar el perfil de resistencia antibiótica de cepas de Salmonella enterica aisladas durante el proceso de beneficio de cerdos en un matadero de Lima Metropolitana. Se utilizaron 148 cepas de Salmonella enterica aisladas de muestras de heces y ganglios mesentéricos de cerdos. La evaluación de la resistencia antibiótica se realizó por el método de difusión de disco (Kirby-Bauer). Se consideraron 13 antibióticos de uso frecuente en medicina humana, de los cuales la mayoría de ellos son usados también en cerdos como profilácticos y promotores de crecimiento. Se encontró que todas las cepas presentaron resistencia hacia la tetraciclina y todas presentaron sensibilidad frente al ciprofloxacino. Así mismo, todas las cepas fueron resistentes a por lo menos un antibiótico.

Palabras clave: Salmonella enterica; cerdos; resistencia antibiótica

\section{Abstract}

The aim of this study was to determine the profile of antibiotic resistance of isolated Salmonella enterica strains during the slaughtering process of pigs in a slaughterhouse in Lima. Strains of Salmonella enterica $(n=148)$ isolated from stool samples and mesenteric ganglia of pigs were used. The evaluation of antibiotic resistance was

${ }^{1}$ Laboratorio de Salud Pública y Salud Ambiental, Facultad de Medicina Veterinaria, Universidad Nacional Mayor de San Marcos, Lima, Perú

${ }^{2}$ Laboratorio de Microbiología y Parasitología Veterinaria, Facultad de Medicina Veterinaria, Universidad Nacional Mayor de San Marcos, Lima, Perú

${ }^{3}$ E-mail:dramosd@unmsm.edu.pe

Recibido: 17 de julio de 2018

Aceptado para publicación: 23 de diciembre de 2018 
performed by the disc diffusion method (Kirby-Bauer). Thirteen antibiotics of frequent use in human medicine were considered, of which most of them are also used in pigs as prophylactic and growth promoters. All strains showed resistance to tetracycline and all showed sensitivity to ciprofloxacin. Likewise, all strains were resistant to at least one antibiotic.

Key words: Salmonella enterica; pig; antibiotic resistance

\section{INTRODUCCIÓN}

La salmonelosis es una enfermedad transmisible por alimentos (ETA) de distribución mundial y de carácter zoonótico, causada por cepas de Salmonella spp. Las cepas de Salmonella spp pueden afectar a diversas especies animales, incluyendo el cerdo, al cual se han vinculado brotes de enfermedad en humanos (Vadillo et al., 2003; Cardoen et al., 2009).

La salmonelosis, después de la campilobacteriosis, ha sido reportada como una zoonosis común en todos los países de la Unión Europea (UE). En 2016 se produjeron 94530 casos confirmados de salmonelosis en humanos, siendo la tasa de notificación en la UE de 20.4 casos por 100000 personas, resultado que fue ligeramente superior a los cuatro años anteriores (20.3-21.9) (EFSA, 2017).

Bahnson et al. (2006) determinaron que el ingreso de cerdos portadores de Salmonella spp a los mataderos en el oeste de Estados Unidos constituye un riesgo de transmisión y la mayor fuente de contaminación es a lo largo de la línea de sacrificio (Lo Fo Wong et al., 2004; Arguello et al., 2012; Gomes-Neves et al., 2012). La diseminación de Salmonella a través de las heces de cerdos es una fuente potencial de contaminación de las canales en el matadero. Asimismo, las cepas ambientales a lo largo de la línea de sacrificio pueden ser también res- ponsables de esta contaminación (GarcíaFeliz et al., 2009; Hernández et al., 2013).

El empleo de los antibióticos en niveles bajos (subterapéuticos) proporcionados en alimentos durante el periodo de destete de los lechones mejora la estabilización de la flora intestinal, reduce la mortalidad y morbilidad por desórdenes gastrointestinales; así mismo, en niveles altos (terapéuticos) son útiles para el tratamiento de enfermedades como neumonías, infecciones intestinales y disentería (Cromwell, 2002; Hofacre et al., 2013). La mayoría de los antibióticos usados son definidos por la Organización Mundial de la Salud como de «importancia crítica» en medicina humana, de allí que su uso en los animales de producción se haya convertido en una fuente de desarrollo de bacterias entéricas resistentes o multirresistentes a estos antibióticos, generando un problema de salud pública, ya que estos microorganismos pueden ser transmitidos a los humanos a través de la cadena alimentaria (Collignon et al., 2009; Cameron, 2016).

El monitoreo de la resistencia antibiótica de Salmonella es importante para determinar la elección del tratamiento contra la salmonelosis en cerdos, así como para identificar cepas resistentes que puede ser transferidas a humanos (Van der Wolf et al., 1999). El objetivo del presente estudio fue determinar el perfil de resistencia antibiótica de cepas de Salmonella enterica aisladas durante el proceso de beneficio de cerdos en un matadero de Lima Metropolitana. 


\section{Materiales y Métodos}

La determinación del perfil de resistencia antibiótica se realizó en el Laboratorio de Salud Pública y Salud Ambiental de la Facultad de Medicina Veterinaria de la Universidad Nacional Mayor de San Marcos, Lima (Perú). Se usaron 148 cepas provenientes de un cepario de Salmonella enterica, las cuales fueron aisladas en el año 2015 de 59 ganglios mesentéricos y 89 hisopados fecales de cerdos faenados en un matadero de Lima Metropolitana. Las cepas se mantuvieron en viales con caldo tripticasa de soya (TSB) y glicerol (15\%) como agente crioprotector y almacenados a temperatura de congelación $\left(-60{ }^{\circ} \mathrm{C}\right)(\mathrm{CLSI}, 2012)$.

Las cepas mantenidas en viales fueron descongeladas a temperatura ambiente, para luego realizar el enriquecimiento y preparación del inóculo. Las cepas fueron cultivadas en $2 \mathrm{ml}$ de TSB e incubadas en estufa a $37^{\circ} \mathrm{C}$ entre 2 y $6 \mathrm{~h}$ hasta alcanzar turbidez, la cual se ajustó a una turbidez de 0.5 en la escala de McFarland, equivalente a una población de $1.5 \times 10^{8} \mathrm{UFC} / \mathrm{ml}$ (INS, 2002).

El perfil de resistencia antibiótica se determinó mediante el método de difusión de discos o técnica de Kirby-Bauer. Las cepas aisladas fueron sembradas en agar MuellerHinton y se enfrentaron a 13 compuestos antibióticos: amoxicilina ( $20 \mu \mathrm{g})$, ampicilina (10 $\mu \mathrm{g})$, cefalexina $(30 \mu \mathrm{g})$, ciprofloxacino $(5 \mu \mathrm{g})$, cloranfenicol $(30 \mu \mathrm{g})$, enrofloxacina $(5 \mu \mathrm{g})$, gentamicina $(10 \mu \mathrm{g})$, lincomicina $(2 \mu \mathrm{g})$, ácido nalidíxico $(30 \mu \mathrm{g})$, neomicina $(30 \mu \mathrm{g})$, nitrofurantoína $(300 \mu \mathrm{g})$, sulfametoxazoltrimetoprim $(1.25 / 23.75 \mu \mathrm{g})$ y tetraciclina $(30 \mu \mathrm{g})$. Los antibióticos fueron elegidos en base a su eficacia clínica, disponibilidad en el mercado nacional e importancia para la vigilancia de la resistencia bacteriana. Las placas se incubaron a $37^{\circ} \mathrm{C}$ por $18-24 \mathrm{~h}$. El halo de inhibición resultante permitió interpretar el grado de resistencia microbiana como cepas como sensibles, intermedias o resistentes (CLSI, 2015).
Se realizó una distribución porcentual de la resistencia de los aislados bacterianos frente a los 13 antibióticos mediante el uso de tablas de frecuencia.

\section{Resultados y Discusión}

Las 148 cepas presentaron resistencia frente a tetraciclina, seguido por el cloranfenicol $(90 \%)$ y nitrofurantoína ( $80 \%$ ), mientras que todas presentaron sensibilidad al ciprofloxacino (Figura 1). Estos resultados son superiores a los descritos por otros investigadores (García-Feliz et al., 2008; Mejía et al., 2008; De Jong et al., 2014; Bonardi et al., 2016; Li et al., 2016), quienes atribuyen la resistencia frente a estos antibióticos al uso continuo en los tratamientos de infecciones $y$, en muchos casos, a que son usados como promotores de crecimiento, dado su libre acceso y disponibilidad en el mercado (Van Duijkeren et al., 2003; García-Feliz et al., 2008; Iwu et al., 2016; Li et al., 2016).

Un hallazgo alarmante en el presente estudio es la existencia de resistencia elevada hacia el cloranfenicol. Este antibiótico está prohibido desde 1994 en la Unión Europea como terapéutico en animales, no sólo por el riesgo de generar resistencia microbiana, sino especialmente debido a que el consumo humano de carne con residuos de este antibiótico puede llegar a producir fetotoxicidad, anemia aplásica, supresión de la medula ósea, mayor riesgo de leucemia infantil y el síndrome del bebé gris (Talero-Pérez et al., 2014).

La resistencia encontrada de las cepas de Salmonella enterica a la amoxicilina $(50 \%)$ y ampicilina (10\%) (Figura 1) fueron inferiores a los hallazgos en otros reportes (Astorga et al., 2007; Bonardi et al., 2016; Calayag et al., 2017; Fois et al., 2017), probablemente debido a que estos antibióticos de la familia de $\beta$-lactámicos no son frecuentemente usados en la alimentación de cerdos. 
La resistencia de $30 \%$ a sulfametoxazol-trimetoprim (Figura 1) fue similar a los reportes de Vico et al. (2011) y Li et al. (2016); sin embargo, se han reportado resistencias más altas (Mateu et al., 2002; De Jong et al., 2014; Iwu et al. 2016). El nivel de resistencia a esta combinación es preocupante ya que el cotrimoxazol (combinación de trimetoprim y sulfametoxazol) es el fármaco comúnmente utilizado para controlar las infecciones oportunistas en personas con VIH positivo.

La resistencia del $80 \%$ hacia nitrofurantoína (Figura 1) fue superior a otros reportes (Ibar et al., 2009; Calayag et al., 2017). El uso de este antibiótico en animales destinados al consumo está prohibido en algunos países (Calayag et al., 2017), debido a que la droga al ser metabolizada produce los metabolitos 3-amino-2-oxazolidinone (AOZ), 1-aminohydantoin (AHD) y semicarbazide (SEM), los cuales persisten en los tejidos y son causantes de mutaciones y cáncer (Vass et al., 2008).

La resistencia de las cepas de Salmonella enterica de $20 \%$ a neomicina y $10 \%$ a gentamicina (Figura 1) fue mayor a los resultados de otros investigadores (Sisak et al., 2006; Vico et al., 2011; De Jong et al., 2014; Li et al., 2016). La resistencia está asociada a enzimas que modifican los grupos amino o hidroxilo de la molécula del antibiótico que impiden la unión del antibiótico al ribosoma y por lo tanto bloquean su actividad antibacteriana. Así se tiene que la resistencia frente a gentamicina es producida por $\mathrm{N}$ acetiltransferasas codificada por el gen AAC(3)-I y la resistencia frente a neomicina es producida por las enzimas modificadoras O-fosfotransferasas, $\mathrm{N}$-acetiltransferasas codificada por APH(3')-II y AAC(2'), respectivamente (Botana et al., 2002; Michael et al., 2006; Van Hoek et al., 2011). Por otro lado, la elevada resistencia a la lincomicina $(70 \%$, Figura 1$)$ se debe probablemente a la presencia de resistencia cruzada con los macrólidos, en especial macrólidos usados como promotores de crecimiento (espiramicina y tilosina) (Botana et al., 2002).

No se presentó resistencia en las cepas de Salmonella enterica hacia el ciprofloxacino, lo cual coincide con otros reportes (Hakanen et al., 2006; Crump et al., 2011; De Jong et al., 2014; DANMAP, 2015). La elevada sensibilidad (100\%) de los aislados de Salmonella enterica a este antibiótico, clínicamente importante para medicina humana, es un resultado positivo desde el punto de vista epidemiológico, puesto que las fluoroquinolonas son antibióticos de primera línea para el manejo del tratamiento de sepsis por gramnegativos (Mølbak et al., 1999; Machado et al., 2003).

En el presente estudio se encontró que todas las cepas de Salmonella enterica aisladas (148/148) fueron resistentes a por lo menos un antibiótico. Esto se debería a la probabilidad de que los animales hayan estado expuestos a estas cepas y que estas han ido adquiriendo resistencia, probablemente, por exposiciones constantes a antibióticos en niveles sub-terapéuticos. Según la Organización Mundial de la Salud, cerca del 80\% del consumo total de antibióticos de importancia médica se da en el sector animal en diversos países, principalmente para estimular el crecimiento en animales sanos (INS, 2018).

En la actualidad, los espectros de resistencia de las cepas MDR (resistencia a más de tres familias de antibióticos importantes en la clínica humana) de las serovariedades de Salmonella se han expandido en los últimos años (FDA, 2013; OMS, 2014; CDC, 2016), ante el uso excesivo de antibióticos en animales de producción y a la transmisión de elementos genéticos entre poblaciones microbianas de los mataderos. Por lo tanto, en países como el Perú, existe la necesidad de establecer un monitoreo continuo en la cadena de suministro y en las granjas para determinar si los antibióticos se están usando de manera adecuada. 


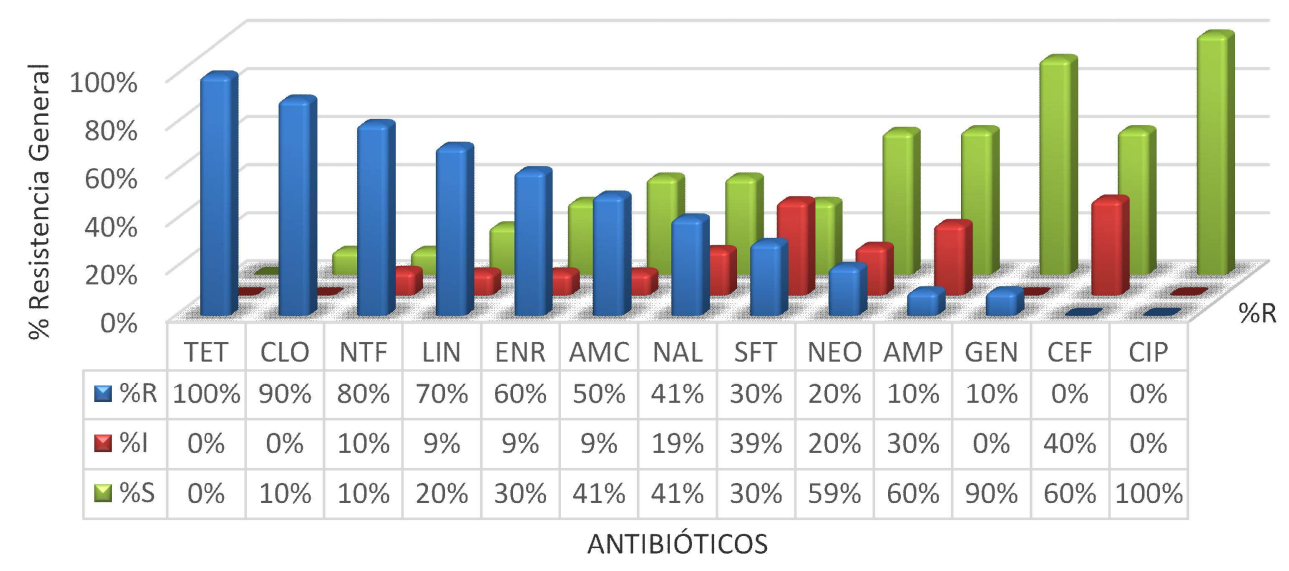

Figura 1. Distribución porcentual de la resistencia y sensibilidad de cepas de Salmonella enterica aisladas de cerdos destinados a consumo en Lima Metropolitana $(n=148)$ frente a 13 antibióticos. AMC: amoxicilina; AMP: ampicilina; CEF: cefalexina; CIP: ciprofloxacino; CLO: cloranfenicol; ENR enrofloxacina; GEN: gentamicina; LIN: lincomicina; NAL: ácido nalidíxico; NEO: neomicina; NTF: nitrofurantoína; SFT: sulfametoxazo; TET: tetraciclina. S: sensible, I: intermedio, R: resistente

\section{ConClusiones}

- Las 148 cepas de Salmonella enterica evaluadas presentaron resistencia frente a tetraciclina.

- Todas las cepas de Salmonella enterica evaluadas fueron resistentes a por lo menos a un antibiótico.

- La alta resistencia hallada frente a la nitrofurantoína es alarmante y debería controlarse su uso en animales de abasto porque produce metabolitos carcinogénicos y mutagénicos.

- Todas las cepas todas presentaron sensibilidad al ciprofloxacino.

\section{Literatura Citada}

1. Arguello H, Carvajal A, Collazos J, García-Feliz C, Rubio P. 2012. Prevalence and serovars of Salmonella enterica on pig carcasses, slaughtered pigs and the environment of four Spanish slaughterhouses. Food Res Int 45: $905-$ 912. doi: 10.1016/j.foodres.2011.04.017

2. Astorga RJ, Echeita A, Maldonado A, Valdezate S, Carbonero A, Aladueña A, Arenas A. 2007. Surveillance and antimicrobial resistance of Salmonella strains isolated from slaughtered pigs in Spain. J Food Protect 70: 1502-1506. doi: 10.4315/0362-028X-70.6.1502

3. Bahnson PB, Fedorka-Cray PJ, Ladely SR, Mateus-Pinilla NE. 2006. Herd-level risk factors for Salmonella enterica subsp. enterica in US market pigs. Prev Vet Med 76: 249-262. doi: 10.1016/j.prevetmed.2006.05.009

4. Bonardi S, Alpigiani I, Bruini I, Barili E, Brindani F, Morganti M, Cavallini P, et al. 2016. Detection of Salmonella enterica in pigs at slaughter and comparison with human isolates in Italy. Int J Food Microbiol 218: 44-50. doi: 10.1016/j.ijfoodmicro.2015.11.005 
5. Botana L, Landoni M, MartínJiménez. 2002. Farmacología y terapéutica veterinaria. España: McGraw Hill Interamericana. $734 \mathrm{p}$.

6. Calayag AM, Paclibare PA, Santos PD, Bautista CA, Rivera WL. 2017. Molecular characterization and antimicrobial resistance of Salmonella enterica from swine slaughtered in two different types of Philippine abattoir. Food Microbiol 65: 51-56. doi: 10.1016/ j.fm.2017.01.016

7. Cameron K. 2016. Evaluación del uso de antimicrobianos como factor de riesgo relacionado con la aparición de resistencia a cefalosporinas en Escherichia coli y Salmonella en cerdos. Tesis Doctoral. Barcelona, España: Universitat Autònoma de Barcelona. $18 \mathrm{p}$.

8. Cardoen S, Van Huffel X, Berkvens D, Quoilin S, Ducoffre G, Saegerman C, Speybroeck N, et al. 2009. Evidence-based semiquantitative methodology for prioritization of foodborne zoonoses. Foodborne Pathog Dis 6: 10831096. doi: 10.1089/fpd.2009.0291

9. [CDC] The Centers for Disease Control and Prevention. 2016. CDC: Resistant Salmonella causes 6200 illnesses a year. University of Minnesota: CIDRAP - Center for Infectious Disease Research and Policy Academic Health Center. [Internet] Available in: http:// www.cidrap.umn.edu/news-perspective/ 2016/12/cdc-resistant-salmonella-causes-6200-illnesses-year

10. [CLSI] Clinical and Laboratory Standards Institute. 2012. Performance standards for antimicrobial disk susceptibility tests. Approved standardEleventh edition. Wayne, PA: CLSI Document M02-A11 Vol 32(1): 11-13, 30.

11. [CLSI] Clinical and Laboratory Standards Institute. 2015. Performance standards for antimicrobial susceptibility testing: Twenty-Fifth Informational Supplement. Wayne, PA: CLSI Document M100-S25 Vol 35(3): 44-51.
12. Collignon P, Powers JH, Chiller TM, Aidara-Kane A, Aarestrup FM. 2009. World Health Organization ranking of antimicrobials according to their importance in human medicine: a critical step for developing risk management strategies for the use of antimicrobials in food production animals. Clin Infect Dis 49: 132-141. doi: 10.1086/599374

13. Cromwell GL. 2002. Why and how antibiotics are used in swine production. Anim Biotechnol 13: 7-27. doi: 10.1081/ ABIO-120005767

14. Crump JA, Medalla FM, Joyce KW, Krueger AL, Hoekstra RM, Whichard JM, Barzilay EJ, et al. 2011. Antimicrobial resistance among invasive nontyphoidal Salmonella enterica in the United States, national antimicrobial resistance monitoring system, 1996-2007. Antimicrob Agents Ch 55: 1148-1154. doi: 10.1128/AAC.01333-10

15. [DANMAP] The Danish Integrated Antimicrobial Resistance Monitoring and Research Programme. 2015. Use of antimicrobial agents and occurrence of antimicrobial resistance in bacteria from food animals, food and humans in Denmark. Denmark: DANMAP. $142 \mathrm{p}$.

16. De Jong A, Smet A, Ludwing $C$, Stephan B, De Graef E, Vanrobaeys M, Haesebrouck F. 2014. Antimicrobial susceptibility of Salmonella isolates from healthy pigs and chickens (2008-2011). Vet Microbiol 171: 298-306. doi: 10.1016/ j.vetmic.2014.01.030

17. [EFSA] European Food Safety Authority. 2017. The European Union summary report on trends and sources of zoonoses, zoonotic agents and foodborne outbreaks in 2016. EFSA J 15: 228 p. doi: $10.2903 /$ j.efsa.2017.5077

18. [FDA]Food and Drug Administration. 2013. Antimicrobial resistance guidances. Maryland: FDA. [Internet]. Available in: https://www.fda.gov/ A n i mal Veterinary/Gui danceComplianceEnforcement/GuidanceforIndustry/ucm123614.htm 
19. Fois F, Piras F, Torpdahl M, Mazza $R$, Consolati S, Spanu C, Scarano C, De Santis E. 2017. Occurrence, characterization, and antimicrobial susceptibility of Salmonella enterica in slaughtered pigs in Sardinia. J Food Sci 82: 969-976. doi: 10.1111/17503841.13657

20. García Feliz C, Collazos JA, Carvajal A, Herrera S, Echeita M, Rubio P. 2008. Antimicrobial resistance of Salmonella enterica isolates from apparently healthy and clinically ill finishing pigs in Spain. Zoonoses Public Hlth 55: 195-205. doi: 10.1111/j.18632378.2008.01110.x

21. García-Feliz C, Carvajal A, Collazos JA, Rubio P. 2009. Herd-level risk factors for faecal shedding of Salmonella enterica in Spanish fattening pigs. Prev Vet Med 91: 130-136. doi: 10.1016/j.prevetmed.2009.05.011

22. Gomes-Neves E, Antunes P, Tavares A, Themudo P, Cardoso MF, Gärtner F, Costa JM, et al. 2012. Salmonella cross-contamination in swine abattoirs in Portugal: carcasses, meat and meat handlers. Int J Food Microbiol 157: 8287. doi: 10.1016/j.ijfoodmicro.2012.04.015

23. Hakanen AJ, Kotilainen P, Pitkänen $S$, Huikko S, Siitonen A, Huovinen P. 2006. Reduction in fluoroquinolone susceptibility among non-typhoidal strains of Salmonella enterica isolated from Finnish patients. J Antimicrob Chemoth 57: 569-572. doi: 10.1093/jac/dk1002

24. Hernández M, Gómez-Laguna J, Luque I, Herrera-León S, Maldonado A, Reguillo L, Astorga R. 2013. Salmonella prevalence and characterization in a free-range pig processing plant: tracking in trucks, lairage, slaughter line and quartering. Int $\mathrm{J}$ Food Microbiol 162: 48-54. doi: 10.1016/j.ijfoodmicro.2012.12.026

25. Hofacre C, Fricke J, Inglis T. 2013. Antimicrobial drug use in poultry. In: Giguère S, Prescott J, Dowling P (eds). Antimicrobial therapy in veterinary me- dicine. $5^{\text {th }}$ ed. USA: John Wiley. p 569586.

26. Ibar MP, Vigo G, Piñeyro P, Caffer MI, Quiroga P, Perfumo C, Centrón D, Giacoboni G. 2009. Serovariedades de Salmonella enterica subespecie enterica en porcinos de faena y su resistencia a los antimicrobianos. Rev Argent Microbiol 41: 156-162.

27. [INS] Instituto Nacional de Salud. 2002. Manual de procedimientos para la prueba de sensibilidad antimicrobiana por el método de disco difusión. Serie de Normas Técnicas N. ${ }^{\circ} 30$. Perú: INS. [Internet]. Disponible en: http:// www.ins.gob.pe/insvirtual/images/ o t r p u b s/pd f/m a n u $1 \%$ 20sensibilidad\%202.pdf

28. [INS] Instituto Nacional de Salud. 2018. Resistencia a los antimicrobianos: Dejemos de administrar antibióticos a animales sanos para prevenir la propagación de la resistencia a los antimicrobianos. Perú: INS. [Internet]. Disponible en: http://antimicrobianos.ins.gob.pe/noticias/265-dejemosde-administrar-antibioticos-a-animalessanos-para-prevenir-la-propagacion-dela-resistencia-a-los-antimicrobianos

29. Iwu CJ, Iweriebor BC, Obi LC, Basson AK, Okoh AI. 2016. Multidrugresistant Salmonella isolates from swine in the Eastern Cape province, South Africa. J Food Protect 79: 1234-1239. doi: 10.4315/0362-028X.JFP-15-224

30. Jensen GM, Lykkesfeldt J, Frydendahl K, Moller K, Svendsen $O$. 2004. Pharmacokinetics of amoxicillin after oral administration in recently weaned piglets with experimentally induced Escherichia coli subtype O149: F4 diarrhea. Am J Vet Res 65: 992-995. doi: 10.2460/ajvr.2004.65.992

31. Li Y, Cai Y, Tao J, Kang X, Jiao Y, Guo R, Wang G, Pan Z, Jiao, X. 2016. Salmonella isolated from the slaughterhouses and correlation with pork contamination in free market. Food Control 59: 591-600. doi: 10.1016/ j.foodcont.2015.06.040 
32. Lo Fo Wong D, Dahl J, Stege H, Van Der Wolf P, Leontides L, Von Altrock A, Thorberg B. 2004. Herd-level risk factors for subclinical Salmonella infection in European finishing-pig herds. Prev Vet Med 62: 253-266. doi: 10.1016/ j.prevetmed.2004.01.001

33. Machado MO, Fabelo CJ, Martínez B. 2003. Ciprofloxacino en el tratamiento ambulatorio. Medicentro 7(4). [Internet]. Disponible en: http://medicentro.sld.cu/index.php/medicentro/article/ view/907/916

34. Mateu EM, Martin M, Darwich L, Mejia W, Frias N, García F. 2002. Antimicrobial susceptibility of Salmonella strains isolated from swine in Catalonia, Spain. Vet Rec 150: 147-150. doi: 10.1136/vr.150.5.147

35. Mejía W, Calatayud M, Zapata D, Quintero A, Sánchez D, Mateu E. 2008. Sensibilidad a los antibióticos de cepas de Salmonella aisladas en granjas porcinas del estado Zulia. Rev Cient 18: 674-681.

36. Michael GB, Butaye P, Cloeckaert A, Schwarz S. 2006. Genes and mutations conferring antimicrobial resistance in Salmonella: an update. Microbes Infect 8: 1898-1914. doi: 10.1016/j.micinf.2005.12.019

37. Molbak K, Baggesen D, Aarestrup F, Ebbesen J, Engberg J, Frydendahl K, Gerner-Smidt P, et al. 1999. An outbreak of multidrug-resistant, quinolone-resistant Salmonella enterica serotype Typhimurium DT104. New Engl J Med 341: 1420-1425. doi: 10.1056/ NEJM199911043411902

38. [OMS] Organización Mundial de la Salud. 2014. Antimicrobial resistance: global report on surveillance 2014. Drug resistance. [Internet]. Available in: http:/ /apps.who.int/iris/bitstream/handle/ 10665/112642/97892415-64748 eng.pdf?sequence $=1$
39. Sisak F, Havlickova H, Hradecka H, Rychlik I, Kolackova I, Karpiskova R. 2006. Antibiotic resistance of Salmonella spp isolates from pigs in the Czech Republic. Vet Med-Czech 51:303-310.

40. Talero-Pérez, YV, Medina OJ, RozoNúñez W. 2014. Técnicas analíticas contemporáneas para la identificación de residuos de sulfonamidas, quinolonas y cloranfenicol. Universitas Scientiarum 19: 11-29. doi: 10.11144/Javeriana.SC19-1.taci

41. Vadillo S, Píriz S, Mateos E. 2003. Manual de microbiología veterinaria. España: McGraw-Hill. 843 p.

42. van der Wolf PJ, Bongers JH, Elbers AR, Franssen FM, Hunneman WA, Van Exsel AC, Tielen MJ. 1999. Salmonella infections in finishing pigs in The Netherlands: bacteriological herd prevalence, serogroup and antibiotic resistance of isolates and risk factors for infection. Vet Microbiol 67: 263-275. doi: 10.1016/S0378-1135(99)00054-1

43. van Duijkeren E, Wannet WJ, Houwers DJ, van Pelt W. 2003. Antimicrobial susceptibilities of Salmonella strains isolated from humans, cattle, pigs, and chickens in the Netherlands from 1984 to 2001. J Clin Microbiol 41: 3574-3578. doi: 10.1128/JCM.41.8.3574-3578.2003

44. van Hoek A, Mevius D, Guerra B, Mullany P, Roberts AP, Aarts H. 2011. Acquired antibiotic resistance genes: an overview. Front Microbiol 2: 1-27. doi: 10.3389/fmicb.2011.00203

45. Vass M, Hruska K, Franek M. 2008. Nitrofuran antibiotics: a review on the application, prohibition and residual analysis. Vet Med-Czech 53: 469-500.

46. Vico JP, Rol I, Garrido V, San Román B, Grilló MJ, Mainar-Jaime RC. 2011. Salmonellosis in finishing pigs in Spain: prevalence, antimicrobial agent susceptibilities, and risk factor analysis. J Food Protect 74: 1070-1078. doi: 10.4315/0362-028X.JFP-10-515 